\title{
ON THE USE OF TIME-FREQUENCY WARPING OPERATORS FOR ANALYSIS OF MARINE-MAMMAL SIGNALS
}

\author{
Cornel Ioana, André Quinquis
}

\author{
ENSIETA, 2 rue François Verny, Brest - FRANCE \\ E-mail : ioanaco@ensieta.fr, quinquis@ensieta.fr
}

\begin{abstract}
Processing marine-mammal signals for passive oceanic acoustic tomography or species classification are problems that have recently attracted attention in scientific literature. Practically, it has been observed that the timefrequency structures of the marine-mammal signals are generally non-linear. This fact dramatically affects the performances achieved by the Cohen's class methods, knowing that these methods correctly perform only for linear time-frequency structures.

Fortunately, it is possible to generate other class of time-frequency representations (TFRs) using the warping operator principle. Based on this principle, we propose a new method for marine-mammal signal characterization. More precisely, many warping operators will be used to "linearize" the time-frequency content of the signal. Furthermore, the chirp-like structures will be estimated. The results obtained for real data illustrate the attractive outlines of the proposed method.
\end{abstract}

\section{INTRODUCTION}

Motivation for processing marine-mammal signals stems from increasing interest in the behavior of endangered marine mammals [1]. The ultimate goal of the current research in this field is to develop tools for analysis of the emitted signal for species identification and monitoring. On the other hand, the characterization of underwater environment is a current topic very challenging. A potential application is the oceanic active tomography [2], which provides an environmental characterization using a man-made emitted signal. Nevertheless, it is possible to imagine the passive tomography concept which will benefit by the generated signals by the natural sources (opportunity sources).

These two applications constitute arguments for the development of some adequate characterization tools of signals emitted by marine-mammals.

In numerous studies [3], and for well-known reasons, the spectrogram [4] has been used as the interface between sound and feature extraction. Nevertheless, in many applications, such as passive sonar signal processing, the spectrogram does not provide enough freedom to the user. On the other hand, as all the Cohen's class members [4], the spectrogram analysis is limited in the case of non-linear time-frequency analysis.

The current technique for analysis of non-linear T-F structures is based on the use of warping operators [5]. In time-frequency analysis context, its application produces the "linearization" of the time-frequency behavior of the signal. The design of warping operators imposes the knowledge of the time-frequency behavior of the signal. Moreover, if the T-F components of the signal have different T-F behavior (example: Hyperbolic Chirps or Linear Chirps), it is necessary to efficiently combine many warping operator types. For these reasons, in order to deal with the passive underwater context, the classical warping operator principle needs to be adapted.

In this work, we propose a new method for timefrequency analysis which is based on signal-dependent selection of some warping operators. The aim is to obtain an efficient time-frequency description of marinemammals emissions. Therefore, the examples will illustrate the performances of this method in a real-data context.

The organization of this paper is as follows. In section 2 we briefly present the warping operator (WO) concept. In section 3 we propose a new method for underwater signal analysis, based on the efficient combination of some time-frequency warping operators. In section 4 we will study the performances of the proposed approach using some real underwater mammals signals. Section 5 "Conclusion" - highlights the significance of the results.

\section{WARPING OPERATOR PRINCIPLE}

Typical time-frequency analysis of signals emitted by marine-mammals involves the use of Cohen's class members such as spectrogram or Wigner-Ville distribution (WVD) [3,4] or wavelet-based methods. While these methods are natural for signals containing pulses, sinusoidals, linear chirping, many other signal classes exist that are not well described in the terms of time, frequency or scale. Matching these types of signals requires a new joint distribution with different instantaneous frequency and group delay localization properties. One of the most known techniques [5] is the unitary similarity transformation. Using this concept, it is possible to construct distributions to match almost any 
one-to-one group delay or instantaneous frequency characteristics. One of the most used unitary transforms is the axis transformation [5], defined for a signal $s(t)$ as an operator $\mathbf{U}$ on $l^{2}(\Re)$, whose effect is given by

$$
(\mathbf{U} s)(x)=\left|w^{\prime}(x)\right|^{1 / 2} s[w(x)]
$$

where $w$ is a smooth, one-to-one function ([5]). Generally, these functions are chosen to ensure the "linearization" of the signal time-frequency behavior. Therefore, for a signal expressed as :

$$
s(t)=e^{j 2 \pi\left(f_{0} t-\beta m(t)\right)}
$$

(where $m(t)$ is the frequency modulation law, $f_{0}$ the central frequency and $\beta$ - the modulation rate), the associated time-warping function is defined as the inverse of modulation function $m(t)$ [6]. For example, for a signal defined as

$$
s(t)=e^{j 2 \pi\left(0.38 t-0.02 t^{1.3}\right)}
$$

the associated warping function can be defined as [6]

$$
w(t)=t^{\frac{1}{1.3}}
$$

In the next figure, the effect of this warping operator, applied on signal $s$, in the time-frequency plane is plotted.
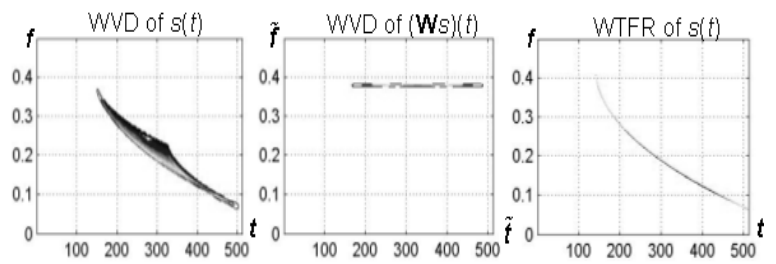

Figure 1. The time-frequency effect of the signal warping

The new time-frequency coordinates are related to the standard ones via [5]

$$
\tilde{t}=w(t) ; \tilde{f}=f \dot{w}_{\alpha}^{-1}(w(t)) \text { - for time warping operator }
$$

where $\dot{w}$ denotes the derivate of $w$. As is exemplified in figure 1 , the effect of warping operator application is the linearization of time-frequency structure of the analyzed signal. This linearization is materialized in the timefrequency plane by the processing of the TFR of the warped signal. Usually, the TFRs of the Cohen's class (CTFR) are used.

Finally, the warped based TFR (WTFR) is obtained by unwarping the coordinates of the CTFR using the following formula [5] :

$$
\operatorname{WTFR}_{s}^{w}(t, f)=\operatorname{CTFR}_{W s}\left(w^{-1}(t), f \dot{w}\left(w^{-1}(t)\right)\right)
$$

The result is a cross-terms free time-frequency distribution, providing a correct concentration of the energy across the theoretical instantaneous frequency law.
This main property of the warping-based TFR is illustrated in the figure 1 for the signal given in (3).

In the case of marine-mammal sounds, many nonlinear time-frequency structures generally occur. The warping-based time-frequency analysis of these types of structures imposes the separation and behavior estimation of each time-frequency component.

One possible method is based on the signal decomposition on an extended basis function dictionary that can accurately represent a multiple T-F structure signal, even for the non-linear case [7]. Nevertheless, in order to achieve good analysis performances, the dictionary size must be large. Consequently, some computational difficulties appear when large number of elementary functions must be processed.

In the next section we propose an alternative method which is based on signal component separation using different warping operators.

\section{WARPING BASED CHARACTERIZATION OF UNDERWATER SIGNALS}

Using the principle previously presented, we propose in this section a warping based method for efficiently characterize the underwater signals. This method is based on the parallel application of many warping operators and, for each issued signal representation, we look for linear time-frequency structures existing in a given timefrequency region of interest. In the most general case, this signal can be expressed as

$$
s(t)=\sum_{C_{i}} \sum_{j=1}^{N_{C_{i}}} s_{j}^{\left(C_{i}\right)}
$$

where $\left\{C_{i}\right\}$ is the set of the time-frequency structure types (classes) and $\left\{s_{j}^{\left(C_{i}\right)}\right\}$ is the set of signal components having a $C_{i}$-type time-frequency behaviour. $C_{i}$ could denote the hyperbolic frequency modulation (HFM) class, $k^{\text {th }}$ order Power Frequency Modulation (K-PFM) [5,6] linear chirp class, etc. $N_{C_{i}}$ is the number of $C_{i}$-type timefrequency structures which composes $s(t)$.

We consider $\left\{\mathbf{W}_{\mathbf{i}}\right\}$ - the family of warping operators associated to the classes $\left\{C_{i}\right\}$. The effect of the application of an element of this family, $\mathbf{W}_{\mathbf{i}}$, on $s(t)$ is the linearization of time-frequency structures corresponding to $\left\{s_{j}^{\left(C_{i}\right)}\right\}$.

$$
S b C_{i}=\left\{\tilde{s}_{j}^{C_{i}}\right\} ; W V D_{\mathbf{w}_{i} c_{j}^{c_{i}}}(t, f) \rightarrow \delta(f-\underbrace{\left(c t+f_{0}\right)}_{\text {chirp }})
$$

Organizing the $\left\{\mathbf{W}_{\mathbf{i}}\right\}$ operators in parallel manner and using the Matching Pursuit-based Chirplet Estimation (MPCE) procedure proposed in [8], we can estimate all the parameters of signal components. This method, called 
Warped-based Signal Decomposition (WSD), is illustrated in the next figure.

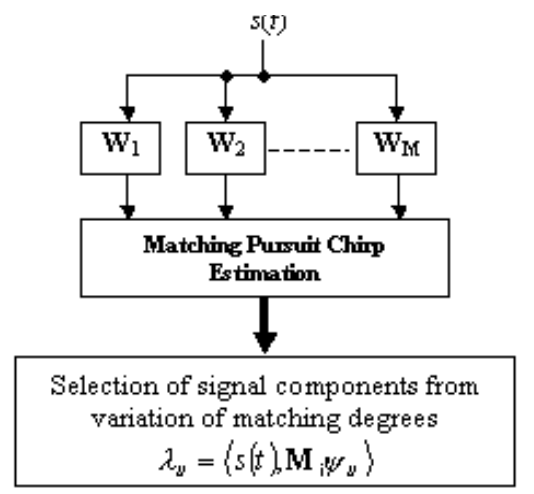

Figure 2. Warping based Signal Decomposition algorithm

After the application of $\mathbf{W}_{\mathbf{i}}$, all the $C_{i}$ time-frequency components of the signal will be linearized (relation (8)) and the others will have arbitrary shapes. Consequently, in each branch, looking for linear T-F structures, the components $\mathbf{W}_{\mathrm{i}} \mathrm{s}(t)$ will be well matched, thanks to their linear T-F behaviour. For the other structures, even if they are more energetic, the chirplet decomposition coefficients will be smaller than the ones corresponding to chirplet structures.

Finally, the estimations obtained from each branch are used to generate, by the corresponding modulation, $\mathbf{M}_{\mathbf{i}}$, the T-F components. Analysing the variation of the correlation degree values $\lambda_{i j}$, (figure 2 ) by a conjugate gradient method, we can estimate the number of components for each time-frequency non-linearity type. At the end, the WSD algorithm provides the signal modelling expressed as :

$$
\widetilde{s}(t)=\sum_{C_{i}} \sum_{j=1}^{\widetilde{N}_{C_{i}}} \lambda_{i j}\left(\mathbf{M}_{\mathbf{i}} \psi_{i j}\right)(t)
$$

The elementary function $\left(\mathbf{M}_{\mathbf{i}} \psi_{i j}\right)(t)$ are completely characterized by its instantaneous frequency law (IFL), given by :

$$
\xi_{i j}(t)=f_{0_{i j}}+\beta_{i j} m_{i}(t) ; t \in\left[t_{0 i j}-\frac{D_{i j}}{2} ; t_{0 i j}+\frac{D_{i j}}{2}\right]
$$

The parameter set, $\bar{\theta}_{i j}=\left[t_{0 i j}, f_{0 i j}, \beta_{i j}, D_{i j}\right]$, contains the estimation of time and frequency centers, chirp rate and signal duration. These estimations are provided by the MPCE algorithm. The function $m_{i}(t)$ is directly related to the $\mathbf{W}_{\mathbf{i}}$ warping operator which allowed to a chirplet function $\psi_{i j}$.

The performing of this algorithm is illustrated on a test signal representing an emission of a long-finned pilot whale (Globicephala melas). For this example, the warping operator battery is composed by some power frequency warping operators, defined as

$$
\mathbf{W}_{\mathbf{t}^{\mathbf{k}}}=t^{1 / k}
$$

where the order $k$ spans the interval 0:0.1:2.

The results obtained on some significant branches $(k=0.1,0.3,0.4,0.5,0.6,0.8)$ are illustrated in the next figure.

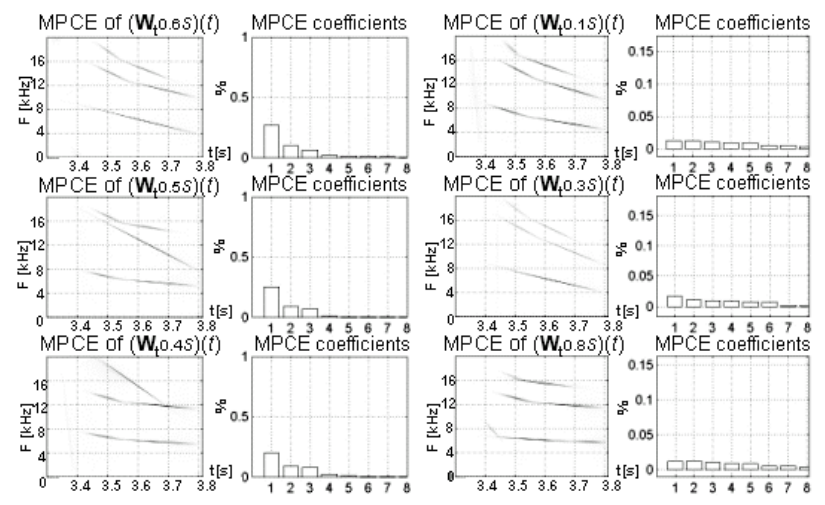

Figure 3. Chirplet estimation of some warped signals

The results of the warped signal approximation by chirplet functions (MPCE) are illustrated. Each warped signal will be approximated by eight chirplet functions using the algorithm proposed in [8]. In the case of 0.6, $0.5,0.4$ power warping orders, we observe that one dominant linear T-F component appears. It is also traduced in the distribution of decomposition coefficients, depicted also in figure 3 . The existence of this dominant chirplet coefficient indicates that it corresponds to the power frequency modulations of order $0.6,0.5$ and 0.4 , respectively. The other chirplet functions, which are less energetic, correspond to different PFM types

For the other warped signals $(\mathrm{k}=0.1,0.3,0.8)$, the decomposition coefficients are energetically reduced. Otherwise, the decomposition coefficients have a reduced dynamic - figure 3). Consequently, the analyzed signal does not contain the assumed non-linear T-F behaviors.

The analyzed signal makes part from a real Globicephala Mellas recording for frequency sampling $\mathrm{F}_{\mathrm{s}}=44.1 \mathrm{kHz}$. The characterization of the complete test signal via the WSD approach is illustrated in the next section.

\section{RESULTS}

In this section, we illustrate the performance of the WSD algorithm in the case of two real marine-mammal emissions. Firstly, we consider a Globicephala Melas observation containing two signals similar to one analyzed in figure 3 (and marked in a dashed rectangle in figure 4). The results are depicted in the next figure. 

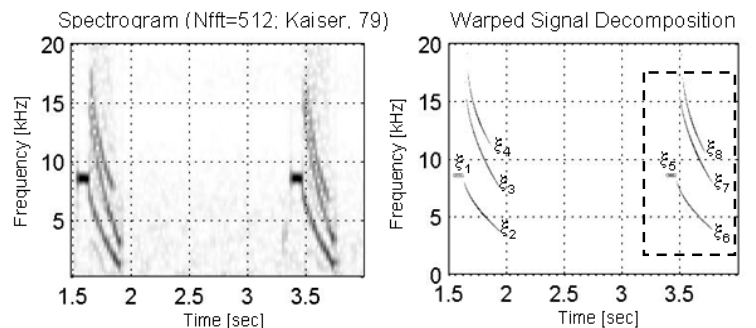

Figure 4. WSD vs spectrogram for Globicephala Melas

As the spectrogram, the WSD provides a visual timefrequency information about the analyzed signal. This TFR is generated by taking the WTFR (relation (6)) of the extracted components. In addition, the WSD furnishes a complementary parametric information (relation (10)) concerning the estimated IFLs. For the analyzed observation, this information is given in table 1 .

Table 1. Estimated parameters of IFLs of Globicephala Melas emission

\begin{tabular}{|c|c|c|c|c|c|}
\hline$\xi$ & $t_{{ }_{0 k}}[\mathrm{sec}]$ & 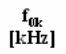 & $\begin{array}{c}\mathrm{D}_{\mathrm{k}} \\
{[\mathrm{sec}]}\end{array}$ & $\beta_{k} F_{s}$ & $m(t)$ \\
\hline$\xi_{1}$ & 1.61 & 8.5 & 0.12 & 0 & \\
\hline$\xi_{2}$ & 1.97 & 4.7 & 0.35 & -0.0054 & $\mathrm{t}^{06}$ \\
\hline$\xi_{3}$ & 1.95 & 10.92 & 0.37 & -0.018 & $t^{05}$ \\
\hline$\xi_{4}$ & 1.9 & 13.2 & 0.35 & -0.035 & $\mathrm{t}^{04}$ \\
\hline$\xi_{5}$ & 3.36 & 8.55 & 0.12 & 0 & - \\
\hline$\varepsilon_{6}$ & 3.62 & 4.82 & 0.35 & -0.0054 & $t^{06}$ \\
\hline$\xi$ & 3.61 & 11.02 & 0.37 & -0.018 & $t^{05}$ \\
\hline$\xi_{8}$ & 3.57 & 13.81 & 0.35 & -0.035 & $t^{04}$ \\
\hline
\end{tabular}

The second test signal is a Dolphin whistle [9]. Using the same structure of the WSD algorithm, we obtain the T-F image given in figure 6. Obviously, the TFR generated by WSD is more accurate than the one provided by the spectrogram. Moreover, WSD T-F image is accompanied by the parametric information, given in table 2.
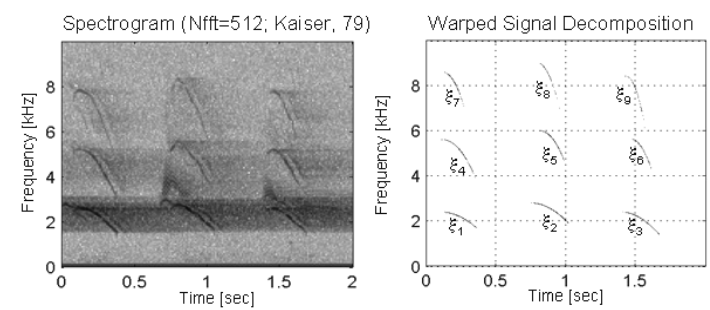

Figure 5. WSD vs spectrogram for Dolphin whistle

Table 2. Estimated parameters of IFLs of Dolphine whistle

\begin{tabular}{|c|c|c|c|c|c|}
\hline$\xi$ & $\mathbf{t}_{\mathbf{i k}}[\mathrm{sec}]$ & 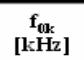 & $\begin{array}{c}\mathrm{D}_{\mathbf{k}} \\
\text { [sec] }\end{array}$ & $\beta_{k} / F_{s}$ & $m(t)$ \\
\hline$\xi_{1}$ & 0.25 & 2.3 & 0.27 & $-9.3 \cdot 10^{-7}$ & $t^{19}$ \\
\hline$\xi_{2}$ & 0.89 & 2.37 & 0.28 & $-10^{-6}$ & $t^{19}$ \\
\hline$\xi_{3}$ & 1.58 & 2.25 & 0.28 & $-2.12 \cdot 10^{-6}$ & $\mathrm{t}^{18}$ \\
\hline$\xi_{4}$ & 0.24 & 4.69 & 0.26 & $-8 \cdot 10^{-7}$ & $\mathrm{t}^{21}$ \\
\hline$\xi_{5}$ & 0.91 & 5.21 & 0.21 & $-6.6 \cdot 10^{-7}$ & $\mathrm{t}^{22}$ \\
\hline$\xi_{6}$ & 1.54 & 4.91 & 0.17 & $-1.61 \cdot 10^{-6}$ & $\mathrm{t}^{21}$ \\
\hline$\xi_{7}$ & 0.2 & 7.66 & 0.18 & $-1.75 \cdot 10^{-6}$ & $t^{21}$ \\
\hline$\xi_{8}$ & 0.87 & 8.11 & 0.16 & $-3.71 \cdot 10^{-6}$ & $t^{2}$ \\
\hline$\xi_{8}$ & 1.52 & 7.73 & 0.13 & $-2.05 \cdot 10^{-6}$ & $t^{22}$ \\
\hline
\end{tabular}

\section{CONCLUSION}

In this paper, we have presented a new method for underwater signal characterization in a passive context. This method is based on the signal warping operation and the estimation, for each deformation, of the virtual linear time-frequency structures. Therefore, the signal modeling (relation (9)) provides the parametric information about the signal. Furthermore, the warping-based TFR of estimated components provides visual information about the analyzed signal.

The examples given for two real-life signals illustrate the outlines of WSD method. The most important is the efficient combination of both visual and parametric information. As observed in figure 4, the T-F images of both spectrogram and WSD indicate that the two T-F regions contain two apparently similar structures. Nevertheless, as indicated in the table 1, the parameters of these components have different values. Therefore, this example proves the capability of WSD to track the variation of T-F parameters. On the other hand, the T-F image issued from WSD, which is noisy-free (see figure 5 ), provides an intuitive and efficient overview of the analyzed phenomenon.

In the future works, we intend to apply the proposed method, as a feature extraction stage, in underwater signal classification. Otherwise, the analytical description of IFLs could be used to adapt the classical underwater signal processing tools in a more realistic concept.

\section{6 . REFERENCES}

[1] W.J. Richardson, C.R. Greene, C.I. Malme, D.H. Thomson, Marine Mammals and Noise, Academic Press, California, 1995.

[2] W. Munk, P. Worcester, C. Wunsch, Oceanic acoustic tomography, Cambridge University Press, 1995.

[3] R.A. Altes, "Detection, estimation and classification with spectrograms", Journal of the Acoustic Society of America, vol. 67, no. 4, pp. 1232-1246, Apr. 1980.

[4] L. Cohen , Time-Frequency Analysis, Pretince Hall, New Jersey, 1993.

[5] R. Baraniuk, "Unitary Equivalence : a new twist on signal processing", IEEE Trans. On Signal Processing, vol. 43, no. 10, October, 1995.

[6] T. Twaroch, F. Hlawatsch, "Modulation and warping operators in joint signal analysis", in Proc. IEEE-SP Int. Sympos. Time-Frequency Time-Scale Analysis, Pittsburgh, PA, pp. 9-12, Oct. 1998.

[7] A. Papandreou-Suppappola, S. Suppappola, "Adaptive timefrequency representations for multiple structures”, Proceedings of the $10^{\text {th }}$ IEEE Workshop on Statistical Signal and Array Processing, Pocono Manor, , pp. 579-583, 2000.

[8] J.C. O'Neill, P. Flandrin, "Chirp Hunting”, IEEE Int. Symp on Time-Frequency and Time-Scale Analysis, pp. 425-428, Pittsburg, Oct. 1998.

[9] www.dolphlinear.com/de_sound. 